\title{
Análise comparativa de rentabilidade: um estudo sobre o Índice de Sustentabilidade Empresarial
}

\section{Profitability comparative analysis: a corporate Sustainability Index Study}

\author{
Elias Hans Dener Ribeiro da Silva ${ }^{1}$ \\ Edson Pinheiro de Lima ${ }^{1,2}$ \\ Sérgio Eduardo Gouvêa da Costa ${ }^{1,2}$ \\ Ângelo Márcio Oliveira Sant'Anna ${ }^{1,3}$
}

\begin{abstract}
Resumo: Organizações têm se esforçado para demonstrar à sociedade suas políticas de boas práticas sociais e ambientais e os investimentos socialmente responsáveis têm sido cada vez mais relevantes, fundamentando a crescente criação de índices de sustentabilidade empresarial. Muitos estudos têm sido feitos sobre o ISE - Índice de Sustentabilidade Empresarial - da BM\&FBovespa, para averiguar se a carteira de ações composta por empresas consideradas sustentáveis traz maior retorno aos acionistas do que a carteira de ações de outros índices. Porém, grande parte dessas pesquisas dá foco ao valor das ações como forma de geração de valor e poucos são os estudos que se pautam em análises fundamentalistas. Este trabalho tem por objetivo comparar indicadores fundamentalistas de rentabilidade entre um grupo de empresas participantes do ISE e um grupo de referência, verificando se a gestão sustentável gera rentabilidade empresarial e a forma como criam valor aos acionistas. Os grupos foram comparados e as evidências encontradas, por meio da utilização do teste estatístico $t$-student, demonstram que, apesar de o ISE possuir uma carteira teórica diferenciada, voltada ao tema social, ambiental e ético, a rentabilidade das empresas que a compõem é semelhante ou inferior à das empresas do grupo de referência. Apesar disso, percebe-se que o grupo ISE apresenta outras formas de criação de valor ao acionista, como menor volatilidade e menor exposição ao risco.

Palavras-chave: ISE; Sustentabilidade; Análise fundamentalista; Valor.
\end{abstract}

\begin{abstract}
Organizations have been striving to show society their policies of good social and environmental practices; consequently, they have been increasingly investing in socially responsible actions, one of the reasons for the growing creation of indexes of corporate sustainability. Many studies have been conducted on the ISE - Corporate Sustainability Index - of BM\&F Bovespa to verify whether the portfolio composed of sustainable companies brings higher return to shareholders than other portfolios of regular companies. However, most of these researches focus on the stock value as a way to generate value, and there are few studies focusing on fundamental analysis. The main purpose of this paper is to compare fundamentalist profitability indicators between a group of companies that compose the ISE index and a reference group, verifying whether sustainable management improves business profitability, and how it creates value for shareholders. The groups were compared; the evidences found by using the $t$-student statistical test show that in spite of having a differentiated theoretical portfolio, focused on social, environmental and ethical issues, the profitability of companies that compose the ISE Group is similar to or lower than that of companies of the reference group. Nevertheless, the results show that the companies of the ISE Group have other ways to create shareholder value, such as lower volatility and risk exposure.
\end{abstract}

Keywords: ISE; Sustainability; Fundamental analysis; Value.

\footnotetext{
${ }^{1}$ Programa de Pós-Graduação em Engenharia de Produção e Sistemas, Pontifícia Universidade Católica do Paraná - PUCPR, Rua Imaculada Conceição, 1155, Escola Politécnica, Bloco 2, $2^{\circ}$ Andar, Prado Velho, CEP 80215-901, Curitiba, PR, Brasil, e-mail: elias.hans@pucpr.br; e.pinheiro@pucpr.br; s.gouvea@pucpr.br; angelo.santanna@pucpr.br

${ }^{2}$ Universidade Tecnológica Federal do Paraná - UTFPR, Av. Sete de Setembro, 3165, Rebouças, CEP 80230-901, Curitiba, PR, Brasil

${ }^{3}$ Escola Politécnica, Universidade de São Paulo - USP, Av. Prof. Luciano Gualberto, Travessa 3, 380, ECEP 05508-010, São Paulo, SP, Brasil
}

Recebido em Nov. 21, 2014 - Aceito em Maio 12, 2015

Suporte financeiro: CAPES (Conselho Nacional de Tecnologia e Desenvolvimento Científico) e CNPq (Coordenação de Aperfeiçoamento de Pessoal de Nível Superior). 


\section{Introdução}

Atualmente as organizações têm se esforçado para demonstrar à sociedade suas políticas de boas práticas sociais e ambientais. Consequentemente, é crescente o número das que publicam relatórios de sustentabilidade seguindo padrões amplamente aceitos, como o GRI - Global Reporting Initiatve - e da indústria financeira, que tem aderido a Princípios para Investimento Responsável que estabelece regras para a seleção de investimentos tendo como critério a conduta das empresas em relação às questões ambientais, sociais e à qualidade das relações com seus stakeholders (Garcia \& Orsato, 2013).

Com isso, investimentos em empresas consideradas sustentáveis têm aumentado significativamente. Hoti et al. (2007) relatam que o crescimento nesse tipo de investimento se deve à menor volatilidade dos índices de sustentabilidade, pois mesmo apresentando retornos médios estatisticamente iguais aos dos fundos convencionais, trazem maior segurança para os investidores em períodos de turbulência na economia.

Essa tendência de criação de índices de sustentabilidade começou na década de 1990, com o primeiro índice de sustentabilidade - Domini 400 Social Index - criado por Kinder, Lydenberg, Domini and Co (MSCI, 2014). Poucos anos depois, em 1999, a Bolsa de Valores de Nova York apresentou o Dow Jones Sustainability Index (DJSI, 2014). Em seguida, diversos outros índices surgiram: em 2001, Londres lançou o FTSE4good (Curran \& Moran, 2007); em 2002, Johanesburgo o Socially Responsible Index (JSE, 2014); e em 2005, em São Paulo, é lançado o Índice de Sustentabilidade Empresarial - ISE - da BM\&FBovespa, a Bolsa de Valores de São Paulo (ISE, 2014).

Em 2014, foi lançada a nona carteira do Índice de Sustentabilidade Empresarial (ISE). A nova carteira reúne ações de 40 companhias. Elas representam 18 setores e somam R $\$ 1,14$ trilhão em valor de mercado, o equivalente a $47,16 \%$ do total do valor das companhias com ações negociadas na BM\&FBOVESPA (BM\&F BOVESPA, 2014b).

Diversos motivos levam as organizações a participarem do processo de seleção de um índice de sustentabilidade. Entre os motivos destacam-se: a captação de recursos financeiros mais vantajosa (McGuire et al., 1988); o acesso ao conhecimento proporcionado pela troca de experiências entre as empresas participantes do grupo (King \& Lenox, 2000); a possível vantagem competitiva como first mover (Christmann, 2000); e, principalmente, o ganho reputacional (Moon \& De Leon, 2007).

Porém, grande parte das pesquisas sobre o ISE dá foco ao valor das ações como elemento para as empresas participarem do grupo de empresas socioambientais, como os estudos apresentados por: Cavalcante et al. (2007), Rezende (2007), Machado, et al. (2009), Beato et al. (2009), Sato et al. (2010), Gomes \&
Tortato (2011). Poucos são os estudos atuais que focam análises fundamentalistas, comparando as empresas que participam do índice e aquelas que não participam.

Este trabalho tem por objetivo apresentar uma comparação entre um grupo de empresas participantes do ISE e um grupo de empresas também de capital aberto, mas que nunca participaram do ISE, baseado em indicadores fundamentalistas de rentabilidade. Tem-se como objetivos específicos verificar se a gestão sustentável pregada pelas empresas gera rentabilidade empresarial significativamente superior à do grupo de empresas que não participam do índice e se geram valor aos acionistas.

\section{Referencial teórico}

Nessa seção, será apresentada uma revisão bibliográfica sobre o desenvolvimento sustentável, com foco nas operações e nos paradigmas econômicos, e sobre a bolsa de valores e os índices de sustentabilidade empresarial, dando ênfase aos indicadores e análises.

\subsection{O valor e a sustentabilidade}

Com as perspectivas de um aumento da população mundial, crescimento do desenvolvimento global e aumento do uso de recursos e seus impactos ambientais associados, está cada vez mais evidente que manter os negócios como de costume não é uma opção para um futuro sustentável (Bocken et al., 2014). O mundo está usando o equivalente a 1,5 planetas para suportar as atividades humanas (WWF, 2012), sendo esse um ritmo insustentável, mesmo em níveis atuais de consumo (Randers, 2012).

A partir de uma visão antropocêntrica, a sustentabilidade é definida como ações e empreendimentos que atendam às necessidades das gerações atuais $\mathrm{e}$ futuras (Laszlo, 2003) e, segundo Bilge et al. (2014), a definição de metas de sustentabilidade mantém ou eleva o sucesso do negócio no longo prazo.

O objetivo básico de qualquer empresa é o sucesso no ambiente de negócios e o aumento de sua participação de mercado. Segundo ITA (2007), para atingir esse objetivo, além de rentabilidade, a integridade ambiental e equidade social são necessárias.

Desde que o conceito de Triple Bottom Line (TBL) foi cunhado por Elkington (1994), as tendências de empresas, considerando o interesse de diferentes stakeholders, têm sido crescentes. O termo "desempenho corporativo" é estendido para incluir não só o aspecto financeiro, mas também aspectos sociais e ambientais. Assim, o desempenho estendido das empresas, muitas vezes chamado de desempenho corporativo sustentável, incluirá componentes de medidas de desempenho financeiro, social e ambiental.

A inclusão desses dois aspectos na medição e avaliação do desempenho organizacional pode 
ser entendida pelo fato de que a responsabilidade da empresa não é apenas para gerar bem-estar econômico (ou seja, lucro), mas também para cuidar da sociedade (pessoas) e do meio ambiente (planeta) (Fauzi et al., 2010).

Neste sentido, construir valor sustentável por meio do TBL cria um valor duradouro para os acionistas e partes interessadas com base em critérios econômicos, ambientais e sociais (Laszlo, 2003).

Essa criação de valor tem evoluído de acordo com os modelos econômicos desde o início da revolução industrial até os mais recentes desenvolvimentos, como a economia de serviços (Buclet, 2011) ou modelos de consumo colaborativo (Botsmann \& Rogers, 2011). Na economia atual, trabalhar apenas com os recursos fundamentais não funciona mais. Organização e marketing devem estar atrelados ao conhecimento e relacionamento (Normann \& Ramirez, 1993). Os modelos econômicos evoluem e fazem os modelos de criação de valor evoluírem com eles, a partir de uma cadeia de valor agregada (Porter, 1979) para uma rede de valor complexa (Allee, 2000).

$\mathrm{Na}$ atual economia baseada em conhecimento, um dos maiores desafios estratégicos é como reconfigurar todo o negócio empresarial, iniciando pelo sistema de criação de valor em si. Os stakeholders são coprodutores de valor e a estratégia organizacional é baseada constantemente na reconfiguração da interação entre os atores (Allee, 2000; Normann \& Ramirez, 1994). A cadeia de valor se transformou em constelação de valor. Normann \& Ramirez. (1993) afirmam que a inovação organizacional é a chave para o sucesso em uma economia incerta e competitiva e define a estratégia corporativa como a arte de criar valor.

Diversos métodos e ferramentas para incorporar a sustentabilidade nas empresas têm sido desenvolvidos nos últimos anos. Considerando esse número crescente de métodos e ferramentas, a identificação e colocação de planos de ação adequados está se tornando cada vez mais complexa (Bovea \& Pérez-Belis, 2012; Pardo et al., 2011). Além disso, a fim de medir as contribuições ambientais e promover a melhoria contínua de circuito fechado, a empresa precisa avaliar como os benefícios de todas as atividades sustentáveis implementadas contribuem para o desenvolvimento global das empresas (Hallstedt et al., 2010).

Esse desenvolvimento por sua vez é resultado dos processos de gestão, desde o planejamento estratégico até sua implementação e está baseado na medição de desempenho corporativa. Assim, o desempenho das empresas refere-se ao resultado final dos processos de gestão em relação às metas corporativas (Fauzi et al., 2010) e é responsabilidade da alta gestão melhorar o desempenho financeiro organizacional uma vez que esse objetivo está entre os grandes interesses dos stakeholders e um alto desempenho financeiro cria oportunidades para melhorar o desempenho social e ambiental, formando assim um ciclo virtuoso (Waddock \& Graves, 1997; Dean, 1988).

\subsection{A bolsa de valores e os índices de sustentabilidade}

O mercado de ações, que é institucionalizado pela bolsa de valores, é conhecido também como mercado de valores mobiliários, no qual os indivíduos negociam ações. Os indivíduos que são chamados de investidores trocam ações, isto é, têm papéis de empresas negociadas nesse mercado. Tais empresas são conhecidas como sociedades por ação (S.A.), com seu capital acionário aberto no mercado de ações brasileiro (Comissão de Valores Mobiliários, 2014).

No Brasil, a bolsa de valores tem o nome de BM\&FBovespa, sendo este o local onde se encontra o centro das negociações do mercado de capitais desenvolvidas pelos investidores brasileiros e as diversas empresas dos mais variados setores da economia brasileira com capital aberto (Dietzold, 2013).

Segundo Dietzold (2013), os índices das bolsas de valores são o conglomerado de empresas que determinadas por setores, tamanho, atuação, liquidez de seus papéis na bolsa, separadas classes possibilitam ao investidor ter uma referência do mercado em que estas empresas atuam. Os índices da bolsa de valores são considerados os termômetros do mercado de ações. Foram criados para possibilitar um maior leque de investimentos orientados a perfis variados de investidores, podendo assim diversificar o investimento ou mesmo alocando o capital naquele que mais convém com sua estratégia ou perfil de investimento.

Diversos índices surgiram e, dentre eles, o Índice de Sustentabilidade Empresarial (ISE), da Bolsa de Valores do Estado de São Paulo que se uniu com a Bolsa de Mercadorias \& Futuros (BM\&F BOVESPA, 2014a). Para que essas empresas participem do ISE, devem participar de um dos níveis de governança corporativa exigidos pela BM\&F BOVESPA (2014b). O maior nível de governança é o Novo Mercado no qual há, somente, empresas com ações ordinárias e com ampla circulação. Esse é o diferencial das empresas listadas no ISE em relação às empresas listadas no IBOVESPA.

O principal objetivo do ISE é proporcionar uma análise do cenário empresarial que possa auxiliar o investidor para tomar decisões compatíveis com as demandas de desenvolvimento sustentável, considerando aspectos de governança corporativa e sustentabilidade empresarial (eficiência econômica, equilíbrio ambiental e justiça social) (BM\&F BOVESPA, 2014b). Desde 2005, empresas listadas 
na BM\&F BOVESPA (2014b), e com as 150 ações de maior liquidez, recebem anualmente um extenso questionário que trata de questões gerais, natureza do produto, governança corporativa e aspectos econômico-financeiros, ambientais e sociais. Para fazer parte da carteira teórica do ISE, as empresas precisam, primeiramente, responder ao referido questionário e atender aos pré-requisitos impostos.

Porém, segundo Souza et al. (2014), o mercado acionário brasileiro é assimétrico quanto à pulverização da propriedade do capital das empresas listadas na BM\&F BOVESPA. É um mercado com cerca de 400 empresas das quais são negociadas duas espécies de ações: ordinárias e preferenciais. É assimétrico porque, nos mercados de bolsas mais desenvolvidos, o número de empresas listadas nas bolsas é muito superior. Pelo mercado versátil americano, por exemplo, existem 2.772 empresas com o capital altamente pulverizado. O Índice de Sustentabilidade Dow Jones (DJSI) é um indicador global e, por isso, tem listadas mais de 318 empresas, distribuídas em 24 países. Com tal magnitude, é possível fazer comparações com o desempenho da Bolsa de Valores de Nova York (Dow Jones). No Brasil, no entanto, essa comparação é muito estreita, pelos motivos supracitados.

\subsection{A caracterização dos indicadores e a análise fundamentalista}

Segundo Pereira \& Urpia (2011), o mercado financeiro é um campo de atuação para os especuladores, investidores que apostam na alta ou baixa de uma determinada ação na bolsa de valores. Os especuladores ganharam espaço e papel singular nos mercados financeiros mundiais, sendo parcialmente responsáveis pela representativa volatilidade em momentos de crises ou de mercados instáveis pelas especulações de queda. Esta forma de investimento aposta na queda ou alta de um determinado papel (ação) sem fundamentos técnicos, manipulando o mercado. Este grupo de investimento é chamado pelos mais conservadores (e contra este modo de investir) de "jogo", deturpando a imagem e a essência da bolsa de valores, pois numa visão clássica do investidor capitalista financeiro o mercado de capitais é um meio criado para o bem do sistema e do progresso das empresas e das economias.

Já os investidores mais conservadores e com visão meramente financeira desenvolvem investimentos com base fundamentalista ou grafista. Os fundamentalistas são aqueles que utilizam balanços contáveis cujas empresas listadas na bolsa de valores são obrigadas a desenvolver e disponibilizar publicamente e, por sua vez, os investidores os analisam para obter informações sobre a empresa e decidir investir ou não, baseados em indicadores de rentabilidade, liquidez, fluxo de caixa, entre outros. Já os investidores grafistas são investidores que analisam os gráficos das empresas para tomar suas decisões, usando ferramentas técnicas e teorias formuladas sobre as tendências dos gráficos desenvolvidos pelas oscilações das ações na bolsa de valores. Estes são os dois tipos de estratégias mais clássicas e utilizadas pelo mercado (Pereira \& Urpia, 2011).

De acordo com Rotella (1992), a análise fundamentalista é inerentemente profética, pois o analista faz projeções que objetivam encontrar equações futuras para as curvas de oferta e demanda do mercado. $O$ fundamentalista frequentemente visa obter o dueto: causa e efeito de uma variável e seus impactos sobre o mercado, ou seja, preocupam-se em definir o motivo pelo qual os preços se direcionarão no futuro.

Segundo Winger \& Frasca (1995), a análise fundamentalista tem em seu alicerce a análise de três fatores: análise da empresa, análise da indústria em que a empresa está inserida e a análise geral da economia (gráfico 2.1). É essencial que estes três fatores sejam analisados, não importando a ordem de suas utilizações

\section{Planejamento da pesquisa}

A pesquisa descrita neste artigo apresenta uma abordagem de pesquisa quantitativa, por analisar dados numéricos, mensuráveis e nenhum subjetivismo na utilização dos métodos estatísticos (Miguel et al., 2012). Quanto à estratégia e método, a pesquisa é caracterizada como uma investigação aplicada, explicativa e histórica por referir-se à resolução de um problema e utiliza fatos já ocorridos, e o pesquisador não pode controlar ou manipular as variáveis estudadas (Vergara, 2007). Além disso, a pesquisa é considerada como sendo longitudinal pelo período estudado e fez-se uso de dados secundários, pois os dados não foram gerados para análise direta desta pesquisa.

Nas seções seguintes, será apresentado o planejamento da pesquisa, assim como a estratégia para coleta de dados, definição das variáveis de estudo e o protocolo de teste.

\subsection{Definição dos grupos}

Para esta pesquisa foram definidos dois grupos de empresas para realização da comparação de desempenho. O primeiro deles é composto por empresas pertencentes ao ISE e o segundo grupo composto por empresas também de capital aberto negociadas na Bolsa de Valores de São Paulo, mas que não participam ou sequer participaram do ISE desde sua criação.

Para definição das empresas que comporão os 2 grupos, foi utilizado um protocolo de pesquisa e tais empresas devem atender a critérios específicos 
previamente definidos. Tais critérios têm por objetivo mitigar erros de comparação e minimizar erros de análises posteriores, buscando apresentar precisão e acurácia nos resultados encontrados.

Apesar de atualmente o Índice de Sustentabilidade Empresarial da BM\&FBOVESPA ser composto por 40 empresas, nem todas foram selecionadas para o estudo, pois, devido às mudanças de composição do índice ano a ano, nem todas atendem aos critérios, que são:

1. Compor o índice de Sustentabilidade Empresarial atual

2. Ter composto o índice nos últimos 5 anos

3. Ter todos os balanços e relatórios publicados no período

Das 40 empresas que compõem o índice atualmente, 29 atendem a todos os critérios e foram selecionadas para o estudo. Tais empresas foram divididas por setores de atuação, conforme classificação encontrada no Software Economatica ${ }^{\circledR}$ System. Posteriormente foi verificado seu valor de mercado ao final do período estudado - dezembro de 2013.

Para formação do segundo grupo (grupo de referência), buscou-se uma composição com empresas que apresentassem a maior similaridade possível com o grupo 1 nos seguintes termos:

- Os dois grupos devem apresentar o mesmo número de empresas em cada setor de atuação;

- Empresas do mesmo setor devem ter o valor de mercado o mais próximo possível.

Porém, para compor o grupo 2, as empresas também devem atender a algumas premissas:

1. Não podem compor o atual índice ISE

2. Não podem ter participado do índice ISE nos últimos 5 anos

3. Devem ter capital aberto e ter ações negociadas na BM\&FBovespa

4. Ter seus balanços e relatórios publicados em todos os anos do período estudado

$\mathrm{Na}$ ausência de empresas que tenham todas as características, serão selecionadas empresas que mais próximo chegarem delas, dando preferência às características "setor de atuação" e "valor de mercado", nessa ordem.

\subsection{Definição das variáveis}

Segundo guia de análise financeira fundamentalista do InfoMoney, um dos mais respeitados jornais financeiros do Brasil, os principais indicadores de rentabilidade permitem avaliar os lucros da empresa em relação a dado nível de vendas, ativos e capital investido. Essa análise vertical é particularmente útil ao comparar o desempenho de uma empresa entre períodos diversos (InfoMoney, 2014). Nesse cenário, os principais indicadores de rentabilidade utilizados para análise são:

- Margem Líquida: determina a porcentagem de cada real de venda que restou após a dedução de todas as despesas, inclusive o imposto de renda. É definida pelo quociente do lucro líquido e da receita líquida de vendas (Fórmula 1). É um indicador semelhante ao de margem operacional, porém, o lucro líquido utilizado para cálculo da margem líquida já inclui o que foi pago em impostos. Segundo Tibúrcio (2012), esse é um indicador de elevado grau de utilidade por ser um índice muito usado para verificar rentabilidade ao acionista.

$$
\text { Margem Líquida }=\frac{\text { Lucro Líquido }}{\text { Receita Líquida de Vendas }}
$$

- Margem EBITDA: em geral, os analistas preferem essa medida ao invés da margem operacional, pois exclui despesas financeiras e depreciação. O EBITDA (Earnings before Interest, Taxes, Depreciation and Amortization) é a expressão em inglês para lucro antes dos impostos, depreciação e amortização. Esse indicador é visto como uma aproximação do impacto das vendas no caixa da empresa, de forma que a margem EBITDA fornece uma ideia de retorno em termos de dinheiro em caixa (Fórmula 2). Além disso, é um número muito valorizado pelo mercado na avaliação de uma empresa porque espelha o desempenho da empresa levando em consideração somente os ganhos gerados por sua atividade principal (Cavalcante \& Associados, 2014).

$$
\text { Margem EBITDA }=\frac{E B T I D A \times 100}{\text { Receita Líquida de Vendas }}
$$

É valido ressaltar que a margem EBTIDA não pode ser calculada para empresas do setor financeiro (bancos, seguradoras, etc.) por essas não possuírem EBITDA válido, pois o balanço financeiro dessas instituições é calculado de forma diferente das demais empresas. A principal causa disso é o cálculo do Provisionamento de Devedores Duvidosos (PDD) que são incluídos na análise contábil de forma diferente dos demais balancetes e tais valores, se confirmados 
como inadimplência, devem ser obrigatoriamente imputados como prejuízos após 1 ano.

- Retorno sobre Patrimônio: O Retorno sobre o Patrimônio Líquido engloba três "alavancas" à disposição da administração para acelerar ou desacelerar uma empresa: a lucratividade; a administração de ativos; e a alavancagem financeira. Ao entender essas três alavancas à disposição da administração, investidores poderão compreender o tipo de Retorno sobre o Patrimônio Líquido que poderão esperar e a capacidade administrativa da empresa (STAFF, 2008). O cálculo pode ser observado na Fórmula 3.

$$
\text { Retorno sobre Patrimônio }=\frac{\text { Lucro Líquido }}{\text { Patrimônio Líquido }}
$$

Desta forma, nesse estudo, os dois grupos de empresas serão comparados sob a ótica dos 3 indicadores apresentados anteriormente.

\subsection{Coleta de dados}

Os dados dos indicadores fundamentalistas analisados nesse estudo foram obtidos com auxílio do software Economatica ${ }^{\circledR}$ System, ferramenta que fornece informações para a análise de ações e fundos. As informações que constituem a base de dados do sistema são oriundas dos demonstrativos financeiros trimestrais e o fechamento diário das ações (Economática, 2014).

Todos os dados foram consultados para o período completo de 2006 a 2013, calculados e consolidados anualmente. Na falta de dados disponíveis no software em relação a algum indicador ou período, esse dado foi tido como missing (*) e tratado como tal para efeito dos testes estatísticos.

\subsection{Protocolo de teste}

Buscando analisar se o grupo de empresas composto por empresas selecionadas e pertencentes ao ISE tem melhor desempenho que o grupo de referência em termos de rentabilidade empresarial, definir-se-á algumas hipóteses para teste:

- Teste 1 - Relacionado ao indicador Margem Líquida

$\mathrm{H}_{0}$ : Não há diferença significativa quanto ao desempenho dos grupos em relação à Margem Líquida

$\mathrm{H}_{1}$ : Há diferença significativa entre o desempenho dos grupos em relação à Margem Líquida
- Teste 2 - Relacionado ao indicador Margem EBITDA

$\mathrm{H}_{0}$ : Não há diferenças significativas quanto ao desempenho dos grupos em relação à Margem EBITDA

$\mathrm{H}_{1}$ : Há diferença significativa entre o desempenho dos grupos em relação à Margem EBITDA

- Teste 3 - Relacionado ao indicador Retorno Sobre Patrimônio

$\mathrm{H}_{0}$ : Não há diferenças significativas quanto ao desempenho dos grupos em relação à Retorno sobre Patrimônio

$\mathrm{H}_{1}$ : Há diferença significativa entre o desempenho dos grupos em relação a Retorno sobre Patrimônio

Para realização da comparação, foi escolhida a aplicação do teste $t$-student de 2 amostras com nível de confiança de $95 \%$. Para tanto, algumas premissas devem ser consideradas e definiu-se o seguinte protocolo de aplicação:

1. Retirar os outliers (máximo 3 rodadas de exclusões);

2. Aplicar "Teste $t$-sudent de 2 amostras" para determinar se há diferença significativa entre os 2 grupos de empresas (teste deve ser realizado separadamente para cada um dos 3 indicadores selecionados);

3. Coletar os resultados gerados pelas iterações do software estatístico;

4. Análise dos resultados.

\section{Resultados e discussão}

Para cálculos estatísticos, foi utilizado o software Minitab $^{\circledR}$, no qual se definiu como default para todos os testes um nível de significância de $95 \%(\alpha=0,05)$. Para melhoramento dos resultados, antes do teste, os dados amostrais passaram por análise para verificar a existência de outliers que pudessem influenciar os resultados. Em todas as amostras foi verificada a presença deles, sendo necessária a remoção. O processo, que tinha em protocolo que poderia ser repetido por no máximo 3 vezes, foi aplicado 2 vezes, até que não fossem mais encontrados outliers, obtendo-se assim "dados amostrais limpos".

Com os dados limpos, foi aplicado o teste $t$-student de 2 amostras para teste de hipóteses, a fim de comparar se havia ou não diferença significativa entre os grupos. Os resultados encontrados nos testes para cada um 
dos indicadores de rentabilidade estudados podem ser verificados nas Tabelas 1,2 e 3.

A Tabela 1 apresenta os principais resultados da comparação entre os grupos em relação ao indicador de margem líquida. Verifica-se que o grupo referência apresenta média de margem líquida maior que a do grupo ISE, porém o P-valor $=0,404(>0,05)$ mostra que com $95 \%$ de confiança pode-se aceitar a hipótese nula $\mathrm{H}_{0}$ e rejeitar a hipótese $\mathrm{H}_{1}$, confirmando que não há diferença significativa entre os grupos.

Por sua vez, a Tabela 2 apresenta os principais resultados da comparação entre os grupos em relação ao indicador de margem EBITDA. Verifica-se que o grupo referência também apresenta uma média de margem EBITDA maior que a do grupo ISE, porém o P-valor $=0,287(>0,05)$ mostra que com $95 \%$ de confiança pode-se aceitar a hipótese nula $\mathrm{H}_{0}$ e rejeitar a hipótese $\mathrm{H}_{1}$, confirmando que também não há diferença significativa entre os grupos em relação às porcentagens de margem líquida obtidas ao final dos períodos.

Os resultados encontrados nos dois primeiros indicadores contradizem algumas pesquisas que relatam que se esperaria uma lucratividade maior para empresas que adotam práticas ambientais, sociais e éticas (Pindle \& Prodhan, 1994; Tsoutsoura, 2004; Lo \& Sheu, 2007; Lo \& Sheu, 2010; El Ghoul et al., 2011).

Por fim, a Tabela 3 apresenta os principais resultados da comparação entre os grupos em relação ao indicador de Rentabilidade do Ativo. Verifica-se que o grupo referência também apresenta média de Retorno sobre patrimônio maior que a do grupo ISE e $\mathrm{P}$-valor $=0,022$ $(<0,05)$ mostra que com $95 \%$ de confiança pode-se rejeitar a hipótese nula $\mathrm{H}_{0}$ e aceitar a hipótese $\mathrm{H}_{1}$, confirmando que há diferença significativa entre os grupos em relação à rentabilidade sobre o patrimônio obtido entre os grupos.

Por meio dos resultados obtidos no estudo, pode-se verificar que o retorno sobre patrimônio obtido pelas empresas do grupo de referência é significativamente superior ao apresentado pelas empresas do grupo ISE. Em relação à margem líquida e da margem EBITDA, o estudo mostrou que não há diferença significativa entre os grupos.

Porém, dois aspectos são percebidos claramente: o grupo de referência nos 3 testes apresentou média

Tabela 1. Indicador de Margem Líquida: GRUPO ISE vs GRUPO REFERÊNCIA.

\begin{tabular}{|c|c|c|c|c|}
\hline & $\mathbf{N}$ & Mean & StDev & SE Mean \\
\hline MARGEM LÍQUIDA GRUPO ISE & 206 & 10,53 & 6,62 & 0,46 \\
\hline MARGEM LÍQUIDA GRUPO REFERÊNCIA & 206 & 11,17 & 8,69 & 0,61 \\
\hline Estimate for difference: & \multicolumn{4}{|c|}{$-0,35$} \\
\hline $95 \%$ CI for difference: & \multicolumn{4}{|c|}{$(-2,132 ; 0,861)$} \\
\hline T-Test of difference $=$ & \multicolumn{4}{|c|}{$0($ vs $\neq):$ T-Value $=-0,83 \mathrm{P}$-Value $=0,404 \mathrm{DF}=383$} \\
\hline
\end{tabular}

Fonte: elaborado pelos autores (2014).

Tabela 2. Indicador de Margem EBITDA: GRUPO ISE vs GRUPO REFERÊNCIA.

\begin{tabular}{|c|c|c|c|c|}
\hline & $\mathbf{N}$ & Mean & StDev & SE Mean \\
\hline MARGEM LÍQUIDA GRUPO ISE & 172 & 24,3 & 10,6 & 0,81 \\
\hline MARGEM LÍQUIDA GRUPO REFERÊNCIA & 172 & 25,6 & 12,6 & 0,96 \\
\hline Estimate for difference: & \multicolumn{4}{|c|}{$-1,34$} \\
\hline $95 \%$ CI for difference: & \multicolumn{4}{|c|}{$(-3,81 ; 1,13)$} \\
\hline T-Test of difference $=$ & \multicolumn{4}{|c|}{$0($ vs $\neq):$ T-Value $=-1,07 \mathrm{P}$-Value $=0,287 \mathrm{DF}=331$} \\
\hline
\end{tabular}

Fonte: elaborado pelos autores (2014).

Tabela 3. Indicador de Rentabilidade sobre Patrimônio: GRUPO ISE vs GRUPO REFERÊNCIA.

\begin{tabular}{lcccc}
\hline & N & Mean & StDev & SE Mean \\
\hline MARGEM LÍQUIDA GRUPO ISE & 227 & 4,49 & 3,6 & 0,24 \\
MARGEM LÍQUIDA GRUPO REFERÊNCIA & 227 & 5,46 & 5,25 & 0,35 \\
& & & $-0,973$ \\
Estimate for difference: & \multicolumn{2}{c}{$(-1,804 ;-0,143)$} \\
95\% CI for difference: & \multicolumn{2}{c}{$0(\mathrm{vs} \neq)$ : T-Value $=-2,30$ P-Value $=0,022$ DF $=400$} \\
T-Test of difference $=$ &
\end{tabular}

Fonte: elaborado pelos autores (2014). 
superior às médias apresentadas pelo grupo ISE. Em compensação, o desvio padrão nos 3 casos também foi maior.

Hoti et al. (2007) argumentam que mesmo apresentando retornos médios estatisticamente iguais aos dos índices convencionais, a volatilidade dos índices de sustentabilidade é menor, representando mais segurança para os investidores em períodos de turbulência na economia.

Esses resultados vão totalmente de encontro aos estudos que relatam que há uma percepção do mercado de que estas empresas estão menos sujeitas a riscos, como apresentado por Barth et al. (1997) e Bansal \& Clelland (2004), e possuem melhor gestão estratégica, sujeitas a menor volatilidade, conforme descrito por Porter \& Linde (1995a, b).

Além disso, segundo Silva et al. (2014), além do retorno financeiro, outras variáveis estão associadas à questão de criação de valor, como pode ser visto na Figura 1.

Ou seja, aspectos ligados à segurança, como a menor volatilidade apresentada pelo grupo ISE por meio de menor desvio padrão, geram valor ao acionista do ponto de vista da mitigação de riscos de longo prazo, mostrando que, apesar de não ter uma média de retorno superior ao grupo de referência, o fato de obter um desvio padrão significativamente inferior ao longo dos anos, mostra constância de resultados. Essa mitigação de riscos está também associada ao paradigma socioeconômico, sob uma perspectiva macro, por empresas assim trazerem mais segurança e proteção aos investidores.

Koehler \& Hespenheide (2013) observam que problemas sociais, de meio ambiente e de governança podem impactar diretamente o desempenho financeiro das empresas por meio de suas operações ou seus produtos, ou indiretamente por meio das ações dos stakeholders ao longo da cadeia de valor, em termos de risco de operações, risco na cadeia de suprimentos e risco dos produtos.

Além disso, não apenas a segurança, mas outros determinantes relacionados à criação de valor estão amplamente relacionados ao grupo de empresas do ISE, por exemplo: valor compartilhado, responsabilidade ambiental, tecnologias mais limpas, transformação social e pensamento enxuto.

Diversos estudos comprovam que a gestão sustentável traz benefícios para o desempenho organizacional no longo prazo (Isaksson \& Steimle, 2009; Kiron et al., 2012; Parisi, 2013).

Kiron et al. (2012) apresenta resultados interessantes em um survey de pesquisa sobre desenvolvimento e implantação de práticas sustentáveis de negócio. A pesquisa, iniciativa conjunta da MIT Sloan Management Review e da Boston Consulting Group, pesquisou mais de 4 mil executivos e gerentes em 113 países. Os resultados apontaram que $70 \%$ das companhias já adotaram permanentemente a sustentabilidade em seus planejamentos estratégicos. Mais de $60 \%$ dos respondentes consideram a sustentabilidade como condição necessária para se manter competitivo no

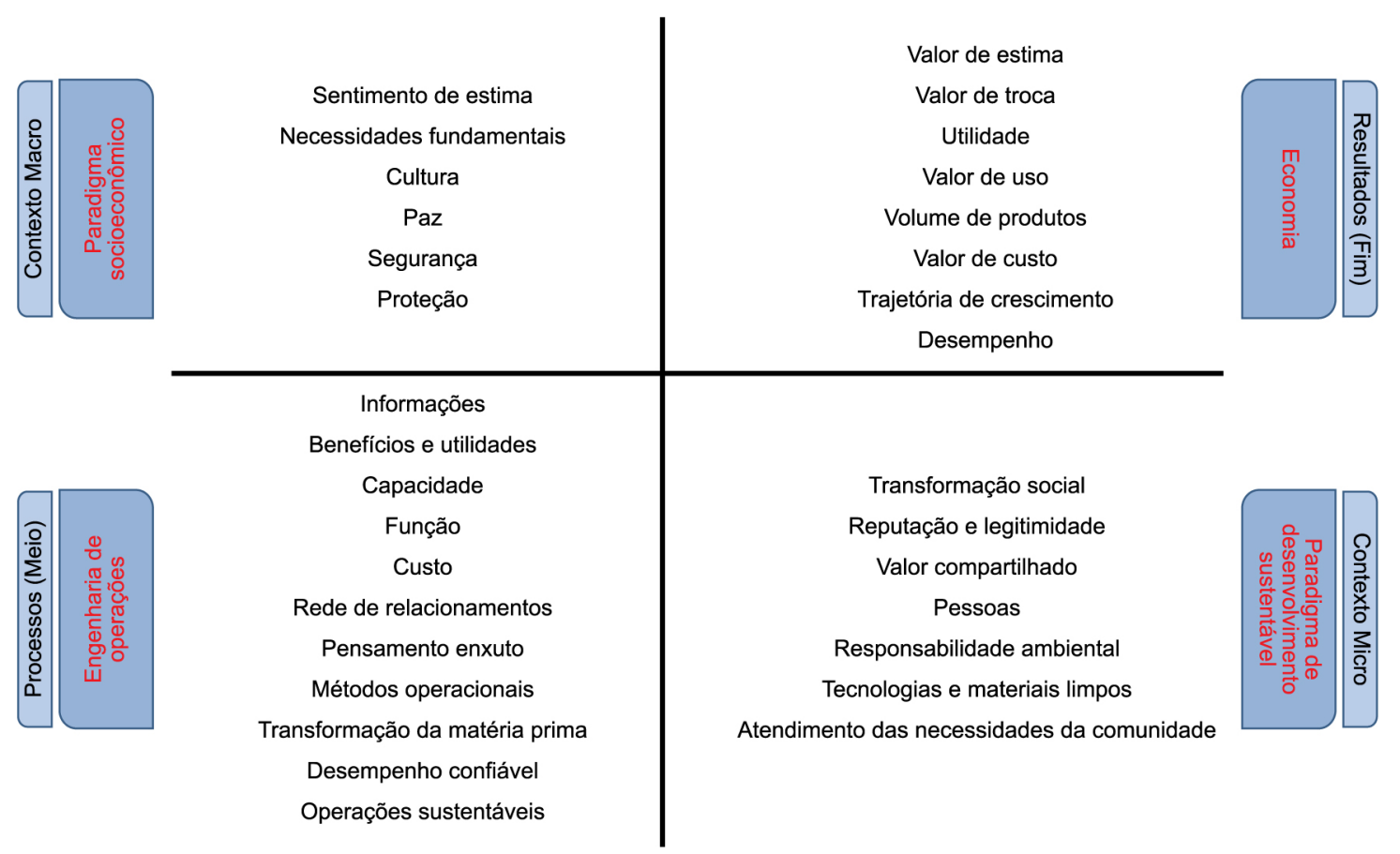

Figura 1. Determinantes para criação de valor. Fonte: Silva et al. (2014). 
mercado. Em análise da pesquisa, identificou-se um grupo ao qual chamaram "Harvesters" (31\% dos respondentes), que estão gerando lucro por meio de suas atividades sustentáveis. Segundo a pesquisa, as empresas estão definitivamente envolvidas com a sustentabilidade e são obrigadas a: combinar a liderança sênior e gestão integrada para o desenvolvimento, baseadas em estratégias sustentáveis; parcerias com organizações que se encontram fora de seus negócios; reconhecer que a adoção de práticas de sustentabilidade leva tempo para aprender e desenvolver competências de gestão sustentável.

Além disso, Bocken et al. (2014) definem um modelo de negócios baseado em três elementos: proposição de valor, criação de valor e captura de valor. A criação de valor é o cerne de qualquer modelo de negócio; empresas normalmente criam valor aproveitando novas oportunidades de negócios, novos mercados e novas fontes de receita (Beltramello et al., 2013; Teece, 2010). Enquanto a proposição de valor é normalmente relacionada com a oferta de produtos e serviços para gerar retorno econômico, em um negócio sustentável a proposição de valor geraria valor ecológico e/ou social mensurável em conjunto com valor econômico (Boons \& Ludeke-Freund, 2013). Assim percebe-se que o retorno financeiro não é a única forma de uma organização criar valor ao acionista e aos demais stakeholders.

\section{Conclusão}

Este trabalho apresentou uma contribuição para a evolução do tema ao utilizar indicadores de desempenho fundamentalistas para análise, fato não encontrado em nenhum dos estudos anteriores de mesma perspectiva. Além disso, os resultados encontrados proporcionaram informações para a realização de uma comparação não apenas em termos de rentabilidade, mas também sob diferentes óticas de criação de valor no contexto organizacional.

Os resultados encontrados no estudo diferem de alguns estudos que a comunidade científica apresentou anteriormente. Sendo assim, 3 hipóteses foram geradas e podem ser estudadas com o intuito de averiguar o desempenho equivalente ou inferior do grupo de empresas do ISE em relação ao grupo de referência, cujas empresas não possuem as mesmas práticas sustentáveis: 1 - o período estudado não foi suficiente para ser considerado longo prazo, fato apresentado como fundamental para a geração de resultados sob a perspectiva sustentável; 2 - as práticas de gestão sustentável estão sendo aprendidas e implementadas há muito pouco tempo e o nível de maturidade convergente com a real gestão sustentável não atingiu seu ponto de equilíbrio; 3 - os resultados podem ter sido influenciados pela recente crise econômica global, impactando ainda mais as empresas do grupo ISE, em sua maioria de grande porte, em que relevante parte de sua receita provém de exportação.

Com isso, sugere-se como trabalhos futuros a replicação do estudo para períodos maiores, caracterizando o longo prazo empresarial, além de buscar a comparação com outros indicadores fundamentalistas, em que, além de indicadores de rentabilidade, deve-se estudar indicadores de estrutura de capital e de liquidez.

Este trabalho também apresentou algumas limitações. A primeira delas é que nem todas as empresas que participam do índice ISE foram utilizadas no estudo. A segunda limitação encontrada foi em relação ao período de estudo, pois, por ser o ISE um índice relativamente recente, impede um estudo com período maior. A terceira limitação foi não terem sido consideradas outras variáveis de análise, como empresas mais afetadas pela crise nos últimos anos. Por fim, a última limitação encontrada foi a comparação ter sido feita apenas em relação a indicadores de rentabilidade.

\section{Agradecimentos}

Os autores gostariam de agradecer ao $\mathrm{CNPq}$ (Conselho Nacional de Tecnologia e Desenvolvimento Científico) o apoio ao projeto de pesquisa por meio da concessão 207871/2012-6 e à CAPES (Coordenação de Aperfeiçoamento de Pessoal de Nível Superior) a concessão de bolsas aos estudantes de pós-graduação.

\section{Referências}

Allee, V. (2000). Reconfiguring the value network. The Journal of Business Strategy, 21(4), 36-39.

Bansal, P., \& Clelland, I. (2004). Talking trash: legitimacy, impression management, and unsystematic risk in the context of the natural environment. Academy of Management Journal, 47(1), 93-103. http://dx.doi. org/10.2307/20159562.

Barth, M. E., Mcnichols, M. F., \& Wilson, P. G. (1997). Factors influencing firms' disclosures about environmental liabilities. Review of Accounting Studies, 2(1), 35-64. http://dx.doi.org/10.1023/A:1018321610509.

Beato, R. S., Souza, T. S., \& Parisotto, I. R. (2009). Rentabilidade dos índices de sustentabilidade empresarial em Bolsa de Valores: um estudo ISE/BOVESPA. Revista de Administração e Inovação, 6(3), 108-127.

Beltramello, A., Haie-Fayle, L., \& Pilat, D. (2013). Why new business models matter for green growth. Paris: OECD Publishing.

Bilge, P., Badurdeenb, F., Seligera, G., \& Jawahirb, I. S. (2014). Model-based approach for assessing value creation to enhance sustainability in manufacturing. In Proceedings of the 47th Conference on Manufacturing Systems (pp. 106-111). Windsor: Procedia CIRP. 
BM\&F BOVESPA. (2014a). BM\&FBOVESPA - A Nova Bolsa. Recuperado em 15 de outubro de 2014, de http:// www.bmfbovespa.com.br.

BM\&F BOVESPA. (2014b). BM\&FBOVESPA divulga a carteira do Índice de Sustentabilidade Empresarial para 2014. Recuperado em 04 de outubro de 2014, de http://www. bmfbovespa.com.br/pt-br/noticias/2013/BMFBOVESPAdivulga-a-carteira-do-ISE-para-2014-2013-11-28. aspx?tipoNoticia $=1$ \&idioma $=$ pt-br.

Bocken, N. M. P., Short, S. W., Rana, P., \& Evans, S. (2014). A literature and practice review to develop sustainable business model archetypes. Journal of Cleaner Production, 65, 42-56. http://dx.doi.org/10.1016/j. jclepro.2013.11.039.

Boons, F., \& Lüdeke-Freund, F. (2013). Business models for sustainable innovation: state-of- the-art and steps towards a research agenda. Journal of Cleaner Production, 45, 9-19. http://dx.doi.org/10.1016/j.jclepro.2012.07.007.

Botsmann, R., \& Rogers, R. (2011). What's mine is yours, how collaborative consumption is changing the way we live. New York: Harper Collins.

Bovea, M. D., \& Pérez-Belis, V. (2012). A taxonomy of ecodesign tools for integrating environmental requirements into the product design process. Journal of Cleaner Production, 20(1), 61-71. http://dx.doi.org/10.1016/j. jclepro.2011.07.012.

Buclet, N. (2011). Le territoire, entre liberté et durabilité. Paris: Presses Universitaires de France.

Cavalcante \& Associados. (2014). Biblioteca de finanças. Construindo e analisando o EBITDA na prática. Recuperado em 28 de outubro de 2014, de http://www. cavalcanteassociados.com.br/index.php?page=financas.

Cavalcante, L. R. M. T., Bruni, A. L., \& Costa, F. J. M. (2007). Sustentabilidade empresarial e desempenho corporativo: uma análise do mercado brasileiro de ações. In Anais do $31^{\circ}$ Encontro Nacional Da Associação Nacional Dos Programas de Pósgraduação em Administração. Rio de Janeiro: ANPAD.

Christmann, P. (2000). Effects of "best practices" of environmental management on cost advantage: the role of complementary assets. Academy of Management Review, 43(4), 663-680. http://dx.doi.org/10.2307/1556360.

Comissão de Valores Mobiliários. (2014). O mercado de valores mobiliários brasileiro (3 ed.). Rio de Janeiro: Comissão de Valores Mobiliários.

Curran, M. M., \& Moran, D. (2007). Impact of the FTSE4Good Index on firm price: an event study. Journal of Environmental Management, 82(4), 529537. http://dx.doi.org/10.1016/j.jenvman.2006.02.010. PMid:16678334.

Dean, K. L. (1988). The chicken and the egg revisited: ties between corporate social performance and the financial bottom line. The Academy of Management Executive, 2, 99-100.
Dietzold, A. (2013). Investidor responsável ou retorno sustentável? Uma análise sobre o ISE - Índice de Sustentabilidade Empresarial (Trabalho de Conclusão de Curso). Universidade Federal de Santa Catarina, Florianópolis.

Dow Jones Sustainability Indices. (2014). DJSI 2014 review results. Recuperado em 8 de outubro de 2014, de http://www.sustainability-indices.com/images/ DJSI_Review_Presentation_09_2014_final.pdf.

Economática. (2014). Recuperado em 1 outubro de 2014, de http://www.economatica.com.br.

El Ghoul, S., Guedhami, O., Kwok, C. C. Y., \& Mishra, D. R. (2011). Does corporate social responsibility affect the cost of capital? Journal of Banking \& Finance, 35(9), 2388-2406. http://dx.doi.org/10.1016/j. jbankfin.2011.02.007.

Elkington, J. (1994). Towards the sustainable corporation: winwin-win business strategies for sustainable corporationable development. California Management Review, 36(2), 90-100. http://dx.doi.org/10.2307/41165746.

Fauzi, H., Svensson, G., \& Rahman, A. A. (2010). Triple bottom line as sustainable corporate performance: a proposition for the future. Sustainability, 2(5), 1345 1360. http://dx.doi.org/10.3390/su2051345.

Garcia, A. S., \& Orsato, R. J. (2013). Índices de sustentabilidade empresarial: porque participar? In Anais do $37^{\circ}$ Encontro da Associação Nacional dos Programas de Pós-Graduação em Administração. Rio de Janeiro: ANPAD.

Gomes, F. P., \& Tortato, U. (2011). Adoção de práticas de sustentabilidade como vantagem competitiva: evidências empíricas. Revista Pensamento Contemporâneo em Administração, 5(2), 33-49.

Hallstedt, S., Ny, H., Robert, K. H., \& Broman, G. (2010). An approach to assessing sustainability integration in strategic decision systems for product development. Journal of Cleaner Production, 18(8), 703-712. http:// dx.doi.org/10.1016/j.jclepro.2009.12.017.

Hoti, S., Mcaleer, M., \& Pauwels, L. L. (2007). Measuring risk in environmental finance. Journal of Economic Surveys, 21(5), 970-998. http://dx.doi.org/10.1111/j.14676419.2007.00526.x.

Índice de Sustentabilidade Empresarial - ISE. (2014). O que é o ISE. Recuperado em 8 de outubro de 2014, de http:// www.isebvmf.com.br/index.php?r=site/conteudo\&id=1.

InfoMoney. (2014). Análise financeira: conheça os indicadores mais usados pelos analistas. Recuperado em 6 de outubro de 2014, de http://www.infomoney.com. br/educacao/guias/noticia/568514/analise-financeiraconheca-indicadores-mais-usados-pelos-analistas.

International Trade Adminitration - ITA. (2007). How does commerce define sustainable manufacturing. Recuperado em 15 de outubro de 2014, de http://www. trade.gov/competitiveness/sustainablemanufacturing/ how_doc_defines_SM.asp. 
Isaksson, R., \& Steimle, U. (2009). What does GRIreporting tell us about corporate sustainability? The TQM Journal, 21(2), 168-181. http://dx.doi. org/10.1108/17542730910938155.

Johannesburg Stock Exchange Limited - JSE. (2014). SRI Index Information: Briefing Document. Johannesburg: JSE.

King, A. A., \& Lenox, M. J. (2000). Industry self-regulation without sanctions: the chemical industry's Responsible Care program. Academy of Management Journal, 43(4), 698-716.

Kiron, D., Kruschwitz, N., Haanaes, K., \& Von Streng Velken, I. (2012). Sustainability nears a tipping point. MIT Sloan Management Review, 53(2), 69-74.

Koehler, D. A., \& Hespenheide, E. J. (2013). Finding the value in environmental, social, and governance performance. Deloitte Review, 12, 97-111.

Laszlo, C. (2003). Sustainable company: how to create lasting value through social and environmental performance. Washington: Island Press.

Lo, S., \& Sheu, H. (2007). Is corporate sustainability a value-increasing strategy for business? Corporate Governance, 15(2), 345-358. http://dx.doi.org/10.1111/ j.1467-8683.2007.00565.x.

Lo, S., \& Sheu, H. (2010). Does corporate sustainability matter to investors? African Journal of Business Management, 4(14), 2856-2863.

Machado, M. R., Machado, M. A. V., \& Corrar, L. J. (2009). Desempenho do Índice de Sustentabilidade Empresarial (ISE) da Bolsa de Valores de São Paulo. Revista Universo Contábil, 5(2), 24-38. http://dx.doi. org/10.4270/ruc.2009211.

McGuire, J. B., Sundgren, A., \& Schneeweis, T. (1988). Corporate social responsibility and financial performance. Academy of Management Journal, 31(4), 854-872. http://dx.doi.org/10.2307/256342.

Miguel, P. A. C., Fleury, A., Nakano, D. N., Turrioni, J. B., Ho, L. L., Morabito, R., Martins, R. A., \& Pureza, V. (2012). Metodologia de pesquisa em engenharia de produção e gestão de operações. Rio de Janeiro: Elsevier.

Moon, S., \& De Leon, P. (2007). Contexts and corporate voluntary environmental behaviors. Organization \& Environment, 20(4), 480-496. http://dx.doi. org/10.1177/1086026607309395.

Morgan Stanley Capital International-MSCI. (2014). MSCI KLD 400 Social Index. Recuperado em 8 de outubro de 2014, de http://www.msci.com/resources/factsheets/ index_fact_sheet/msci-kld-400-social-index.pdf.

Normann, R., \& Ramirez, R. (1993). From value chain to value constellation: designing interactive strategy. Harvard Business Review, 71(4), 65-77.

Normann, R., \& Ramirez, R. (1994). Designing interactive strategy: from value chain to value constellation. Hoboken: John Wiley \& Sons.
Pardo, R. J. H., Brissaud, D., \& Zwolinski, P. (2011). Contribution to the characterization of eco-design projects. International Journal of Sustainable Engineering, 4(4), 301-312.

Parisi, C. (2013). The impact of organisational alignment on the effectiveness of firms' sustainability strategic performance measurement systems: an empirical analysis. Journal of Management \& Governance, 17(1), 71-97. http://dx.doi.org/10.1007/s10997-012-9219-4.

Pereira, E. J. A., \& Urpia, A. G. B. C. (2011). Hipótese dos mercados eficientes vis-à-vis incerteza, convenção e especulação: por uma mudança de paradigma nos mercados financeiros. Pesquisa e Debate, 22(1), 135-155.

Pindle, A. R., \& Prodhan, B. (1994). Ethical conflicts in finance. Oxford: Blackwell.

Porter, M. E. (1979). How competitive forces shape strategy. Harvard Business Review, 57(2), 137-145.

Porter, M., \& Linde, V. D. (1995a). Toward a new conception of the environmental-competitiveness relationship. The Journal of Economic Perspectives, 9(4), 97-118. http:// dx.doi.org/10.1257/jep.9.4.97.

Porter, M., \& Linde, V. D. (1995b). Green and competitive. Harvard Business Review, 73(5), 120-134.

Randers, J. (2012). 2052: a global forecast for the next forty years. Vermont: Chelsea Green Publishing.

Rezende, I. (2007). Um estudo sobre o desempenho financeiro do Índice BOVESPA de Sustentabilidade Empresarial. In Anais do $31^{\circ}$ Encontro da Associação Nacional dos Programas de Pós-Graduação em Administração. Rio de Janeiro: ANPAD.

Rotella, R. P. (1992). The elements of successful trading. New York: Institute of Finance Simon \& Schuster.

Sato, K. H., Silva, W., Nogas, P., \& Yamashiro, A. (2010). Sustentabilidade e responsabilidade social: análise do desempenho do índice de sustentabilidade empresarial. Perspectivas Contemporâneas, 5, 157-177.

Silva, E. H. D. R., Lima, E. P., \& Costa, S. E.G. (2014). Qual o significado de valor? Uma abordagem baseada em diferentes perspectivas [Working Paper]. Curitiba: Pontifícia Universidade Católica do Paraná.

Souza, F., Zucco, A., Tomé, I., \& Pereira, R. (2014). Análise do índice de sustentabilidade empresarial - ISE: um estudo exploratório comparativo com o Ibovespa. Conexxio, 4, 145-159.

Staff, M. F. (2008). Return on equity: an introduction. Recuperado em 28 de outubro de 2014, de http://www. fool.com/investing/beginning/return-on-equity-anintroduction.aspx.

Teece, D. (2010). Business models, business strategy and innovation. Long Range Planning, 43(2-3), 172-194. http://dx.doi.org/10.1016/j.lrp.2009.07.003. 
Tiburcio, C. (2012). Margem líquida. Recuperado em 6 de outubro de 2014, de http://www.contabilidade-financeira. com/2012/03/margem-liquida.html.

Tsoutsoura, M. (2004). Corporate social responsibility and financial performance. Berkeley: University of California at Berkeley.

Vergara, S. C. (2007). Projetos e relatórios de pesquisa em administração. São Paulo: Atlas.

Waddock, S. A., \& Graves, S. M. (1997). The corporate social performance-financial performance link. Strategic
Management Journal, 18(4), 303-319. http://dx.doi. org/10.1002/(SICI)1097-0266(199704)18:4<303::AIDSMJ869>3.0.CO;2-G.

Winger, B., \& Frasca, R. (1995). Investments: introduction to analysis and planning, London: Macmillan.

World Wide Fund for Nature - WWF. (2012). Living planet report 2012. Gland: WWF International. Recuperado em 15 outubro de 2014, de http://awsassets.panda.org/ downloads/1_lpr_2012_online_full_size_single_pages_ final_120516.pdf. 